\title{
Feasibility and Effectiveness of Direct Puncture and Onyx Embolization for Transverse Sinus Dural Arteriovenous Fistula
}

\author{
Taek-kyun Nam ${ }^{1}$ Jun Soo Byun ${ }^{2}$, Hyun Ho Choi ${ }^{1,3}$, Mi Sun Chung ${ }^{2}$, and Eun Jung Lee ${ }^{2}$ \\ Departments of ${ }^{1}$ Neurosurgery and ${ }^{2}$ Radiology, Chung-Ang University Hospital, Chung-Ang University College of Medicine, Seoul; \\ ${ }^{3}$ Department of Neurosurgery, Kangwon National University School of Medicine, Chuncheon, Korea.
}

\begin{abstract}
Direct puncture and embolization of the transverse sinus (TS) for treatment of dural arteriovenous fistula (DAVF) is typically performed with coils with or without glue. We report a case of DAVF at the left TS that was treated with Onyx embolization via direct puncture of the TS. A 75-year-old woman presented with tremor, festinating gait, and dysarthria. A left TS-DAVF with retrograde superior sagittal sinus and cortical venous reflux (Cognard type IIa $+b$ ) was identified on cerebral angiography, and both TSs were occluded with thrombi. We considered that achieving complete cure by transvenous embolization via the femoral vein or transarterial embolization via occipital feeders would be difficult. Thus, we performed a small craniotomy at the occipital bone to puncture the TS. The midportion of the TS was directly punctured with a 21-G microneedle under fluoroscopic guidance. We inserted a 5-F sheath into the TS. A microcatheter was then navigated into the affected sinus. Coils were placed through the microcatheter to support Onyx formation by reducing the pressure of shunting flow. Onyx embolization was performed with the same microcatheter. The DAVF was almost completely occluded except for the presence of minimal shunting flow to the proximal TS. After 1 week, time-of-flight magnetic resonance angiography showed complete resolution of DAVF. The patient showed resolved tremor and markedly improved mental status at 1-month follow up. Direct puncture and embolization of the TS using coils and Onyx is effective and feasible method for the treatment of DAVF when other approaches seem difficult.
\end{abstract}

Key Words: Dural arteriovenous fistula, embolization, Onyx, coil, transcranial

\section{INTRODUCTION}

Dural arteriovenous fistula (DAVF) is responsible for $10-15 \%$ of intracranial arteriovenous abnormalities. ${ }^{1}$ DAVF of the transverse sinus (TS) with cortical venous reflux poses a high hemorrhage risk of $10-40 \%$ and significant morbidity and mortality. ${ }^{2,3}$ DAVF treatment is aimed at reducing the risk of hemorrhage by obliterating the arteriovenous shunting that leads to venous hy-

Received: July 3, 2019 Revised: August 9, 2019

Accepted: August 21, 2019

Corresponding author: Jun Soo Byun, MD, PhD, Department of Radiology, ChungAng University Hospital, 102 Heukseok-ro, Dongjak-gu, Seoul 06973, Korea.

Tel: 82-2-6299-2643, Fax: 82-2-6263-1557, E-mail: flightdr61@hanmail.net

-The authors have no potential conflicts of interest to disclose.

(C) Copyright: Yonsei University College of Medicine 2019

This is an Open Access article distributed under the terms of the Creative Commons Attribution Non-Commercial License (https://creativecommons.org/licenses/ by-nc/4.0) which permits unrestricted non-commercial use, distribution, and reproduction in any medium, provided the original work is properly cited. pertension. Transarterial or transvenous embolization is sometimes not applicable because the venous access can be prevented by sinus thrombosis. A direct surgical approach can be an alternative if percutaneous access is not feasible. Several authors have reported on direct puncture and sinus embolization using coils with or without glue and coil with transarterial Onyx injection for the treatment of transverse-sigmoid sinus DAVF. ${ }^{1,4-9}$ However, to our knowledge, there is no report of direct puncture and sinus embolization using Onyx. We report a case of coil and Onyx embolization after direct cannulation of the TS for the treatment of left TS DAVF.

\section{CASE REPORT}

A 75-year-old woman presented with tremor, festinating gait, and dysarthria. A left-sided TS-DAVF was seen on computed tomography angiography. Digital subtraction angiography con- 
firmed the presence of a Borden II/Cognard IIa+b left TS-DAVF with bilateral TS occlusion (Fig. 1). Given the presence of cortical venous reflux, treatment was recommended.

We deemed that transvenous embolization via the femoral vein would be difficult to perform because both TSs were occluded by thrombi. Moreover, achieving complete cure by transarterial embolization via occipital feeders would be difficult because of numerous fine fistulas in the left TS fed by numerous transosseous fine feeders of both occipital arteries, as well as the left stylomastoid and posterior auricular arteries.

\section{Treatment}

A surgical procedure for accessing TS was performed in the operating room under general anesthesia. The patients were placed in the supine position with right-sided rotation of the upper body and the head. A linear vertical incision was made, and a small craniotomy (size, $2 \mathrm{~cm}$ ) was performed at the midportion of the left TS. The location of the TS was confirmed with an intraoperative Doppler.

Sinus catheterization was performed after the patient had been transferred to the angiography room. Under roadmap guidance, the left TS was directly punctured using a $21-G$ micropuncture needle. A 5-F micropuncture sheath was advanced over a micropuncture wire after needle removal. A 018 Rebar microcatheter (Medtronic Inc., Irvine, CA, USA) was then advanced

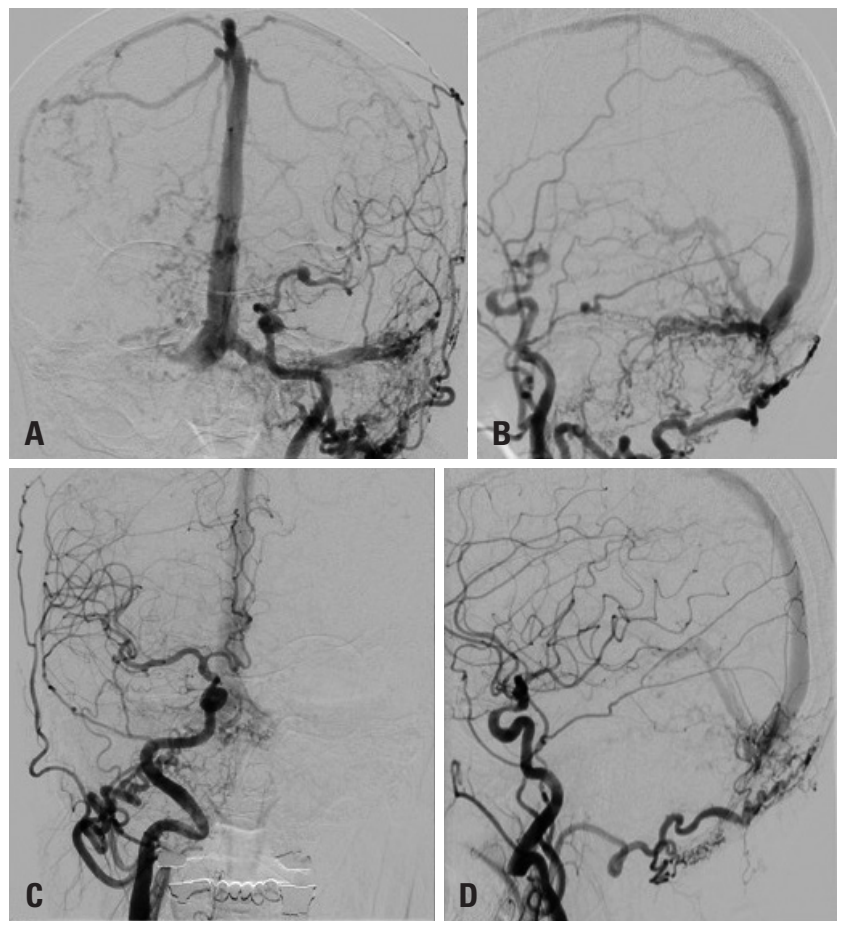

Fig. 1. Initial angiogram. (A and $B)$ Anterior $(A)$ and lateral $(B)$ images of the left CCA angiogram show dural arteriovenous fistula with retrograde flow and cortical venous reflux (Cognard classification lla+b). Diffuse numerous shunting flows are noted in the left transverse sinus. Bilateral sigmoid sinus is occluded. (C and D) Anterior (C) and lateral (D) images of right CCA angiogram show shunting flow into the torcular region. CCA, common carotid artery. over a microwire through the 5-F sheath into the distal end of the left TS. Seven detachable coils were inserted at the distal end of the left TS to reduce pressure of shunting flow through fistulas. After three coils were inserted, a coil frame was intentionally made with a fourth coil proximal to the microcatheter tip, and three more coils were filled into the proximal fourth coil frame (i.e., the microcatheter tip was located at the mid-portion of the coil mass that helps forward the expansion of Onyx). Onyx embolization was performed carefully flowing backward over the puncture site from the proximal occluded point of the left TS to just before the torcula. Final angiography revealed almost complete occlusion of the fistula, except for the presence of minimal shunting flow in the left proximal TS (Fig. 2A-F). The micropuncture sheath was removed from the TS, and bleeding was controlled with hemostatic materials (Floseal ${ }^{\circledR}$, Baxter, Hayward, CA, USA). Bleeding was easily controlled because Onyx flowed backward over the puncture site. A miniplate was fixed over the craniotomy site, and the wound was closed. No complications occurred during the procedure. The patient was monitored overnight in the neurointensive care unit and discharged home 3 days later.

After 1-week follow up, time-of-flight magnetic resonance angiography showed complete resolution of residual shunting in the proximal TS (Fig. 2G-I). At 1-month follow up, tremor, festinating gait, and dysarthria had resolved, and the patient showed markedly improved mental status. We planned follow-up DSA
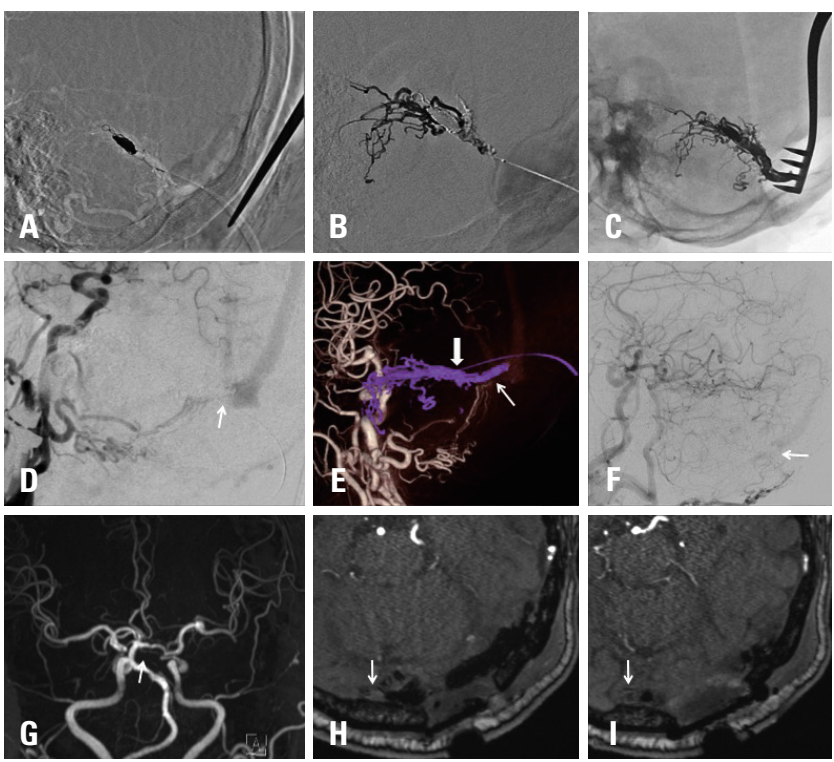

Fig. 2. Treatment and follow up. (A) Lateral image shows inserted coil mass. (B) Lateral image shows Onyx cast during embolization. (C) Lateral image shows final Onyx cast. (D and E) Left three-dimensional common carotid artery angiogram (D) and volume rendering image (E) show residual fistula from the left occipital artery in the proximal TS (arrows). Onyx refluxed over the puncture point in the left TS (thick arrow). (F) Right brachiocephalic angiogram depicts residual fistula from the right occipital artery in proximal TS (arrow). (G-I): 1-week follow-up time-of-flight magnetic resonance angiography depicts the resolution of the fistula in the proximal TS (arrows). TS, transverse sinus. 
and further embolization of the DAVF if the shunt remained.

The patient agreed to use of her clinical and private data being used in research, publications, sharing and archiving.

\section{DISCUSSION}

In this case report, we illustrate the feasibility and effectiveness of both surgical craniotomy and sinus embolization using Onyx for the treatment of TS-DAVF with bilateral TS occlusion. Transcranial access was first described by Mickle and Quisling in 1986. ${ }^{10}$ Several reports have described the use of the transcranial route for direct venous sinus cannulation. Most of the authors embolized venous sinus using coils after direct puncture. ${ }^{1,4-9}$ Some patients were pretreated with transarterial embolization to reduce arterial inflow before the procedure. ${ }^{5,11,12}$ This is the first case using transvenous coil and Onyx embolization after direct puncture of the venous sinus. The advantages of this method are that transvenous coil embolization reduces arterial inflow into the sinus, and complete sinus occlusion can be obtained following Onyx embolization. Onyx showed better closure rates in the treatment of DAVF than glue as a result of better penetration throughout the fistula. ${ }^{13}$ The cohesive liquid form of Onyx is modeled by blood flow. ${ }^{14,15}$ It may flow toward retrograde cortical veins because the flow direction is toward cortical veins and may not move into normal cortical veins because the flow direction is toward the sinus that pushes Onyx away. ${ }^{16}$

The locations of sinus punctures can vary. In all previous reports, the puncture site was proximal to the fistulous segment (i.e., not in the fistulous segment), and the authors used coils only or coils with glue for the embolization of the directly punctured sinus. In our patient, we punctured the midportion of the left TS (i.e., in the fistulous segment) and embolized it from the distal end of the occluded TS with detachable coils and Onyx. Onyx flowed backward over the puncture site in the left TS and stopped just before torcula. The advantage of this puncture location was that it reduced the possibility of massive sinus bleeding after microsheath removal. There was no difficulty with removing the microcatheter even though the microcatheter was captured by Onyx, because the distance between the catheter tip and puncture site was very short, compared to that observed with the transfemoral venous approach.

A direct approach to the sinus should be considered if a transfemoral approach is not feasible in patients for whom transvenous embolization is preferred. Using coils and Onyx embolization may be a feasible and effective combination for the treatment of DAVF with sinus occlusion by direct access.

\section{AUTHOR CONTRIBUTIONS}

Conceptualization: Jun Soo Byun and Taek-Kyun Nam. Data curation: Hyun Ho Choi. Formal analysis: Hyun Ho Choi. Investigation: Mi Sun Chung and Eun Jung Lee. Project administration: Taek-kyun
Nam. Resources: Taek-kyun Nam and Hyun Ho Choi. Supervision: Jun Soo Byun. Validation: Jun Soo Byun and Taek-kyun Nam. Visualization: Mi Sun Chung and Eun Jung Lee. Writing_original draft: Taek-kyun Nam. Writing—review \& editing: Jun Soo Byun.

\section{ORCID iDs}

Taek-kyun Nam ～https://orcid.org/0000-0003-3151-631X Jun Soo Byun https://orcid.org/0000-0003-3210-9505

Hyun Ho Choi https://orcid.org/0000-0003-1170-6829

Mi Sun Chung https://orcid.org/0000-0003-1141-9555

Eun Jung Lee https://orcid.org/0000-0002-2933-2995

\section{REFERENCES}

1. Houdart E, Saint-Maurice JP, Chapot R, Ditchfield A, Blanquet A, Lot $\mathrm{G}$, et al. Transcranial approach for venous embolization of dural arteriovenous fistulas. J Neurosurg 2002;97:280-6.

2. Cognard C, Gobin YP, Pierot L, Bailly AL, Houdart E, Casasco A, et al. Cerebral dural arteriovenous fistulas: clinical and angiographic correlation with a revised classification of venous drainage. Radiology 1995;194:671-80.

3. Duffau H, Lopes M, Janosevic V, Sichez JP, Faillot T, Capelle L, et al. Early rebleeding from intracranial dural arteriovenous fistulas: report of 20 cases and review of the literature. J Neurosurg 1999; 90:78-84.

4. Bruneau M, Lubicz B, Pirotte B, Taib NO, Wikler D, Brotchi J, et al. Selective image-guided venous sinus exposure for direct embolization of dural arteriovenous fistula: technical case report. Surg Neurol 2008;69:192-6.

5. Endo S, Kuwayama N, Takaku A, Nishijima M. Direct packing of the isolated sinus in patients with dural arteriovenous fistulas of the transverse-sigmoid sinus. J Neurosurg 1998;88:449-56.

6. Liu JK, Choudhry OJ, Barnwell SL, Delashaw JB Jr, Dogan A. Single stage transcranial exposure of large dural venous sinuses for surgically-assisted direct transvenous embolization of high-grade dural arteriovenous fistulas: technical note. Acta Neurochir (Wien) 2012;154:1855-9.

7. Zink WE, Meyers PM, Connolly ES, Lavine SD. Combined surgical and endovascular management of a complex posttraumatic dural arteriovenous fistula of the tentorium and straight sinus. J Neuroimaging 2004;14:273-6.

8. Caplan JM, Kaminsky I, Gailloud P, Huang J. A single burr hole approach for direct transverse sinus cannulation for the treatment of a dural arteriovenous fistula. BMJ Case Rep 2014;2014: bcr2013011011.

9. Matsuzaki J, Kono K, Umesaki A, Kashimura Y, Matsumoto H, Terada T. [Transvenous embolization by direct puncture of the superior sagittal sinus using indocyanine green (ICG) videoangiography for treatment of dural arteriovenous fistula of the transversesigmoid sinus: a case report]. No Shinkei Geka 2017;45:591-8.

10. Mickle JP, Quisling RG. The transtorcular embolization of vein of Galen aneurysms. J Neurosurg 1986;64:731-5.

11. Zhang Y, Li Q, Huang QH. Embolization of a superior sagittal sinus dural arteriovenous fistula under intrasinus balloon protection: a case report. Interv Neuroradiol 2015;21:94-100.

12. Watanabe J, Maruya J, Nishimaki K, Ito Y. Onyx removal after embolization of a superior sagittal sinus dural arteriovenous fistula involving scalp artery. Surg Neurol Int 2016;7(Suppl 14):S410-4.

13. Rabinov JD, Yoo AJ, Ogilvy CS, Carter BS, Hirsch JA. ONYX versus n-BCA for embolization of cranial dural arteriovenous fistulas. J 
Neurointerv Surg 2013;5:306-10.

14. Akin ED, Perkins E, Ross IB. Surgical handling characteristics of an ethylene vinyl alcohol copolymer compared with N-butyl cyanoacrylate used for embolization of vessels in an arteriovenous malformation resection model in swine. J Neurosurg 2003;98:36670 .

15. Murayama Y, Viñuela F, Ulhoa A, Akiba Y, Duckwiler GR, Gobin
YP, et al. Nonadhesive liquid embolic agent for cerebral arteriovenous malformations: preliminary histopathological studies in swine rete mirabile. Neurosurgery 1998;43:1164-75.

16. Torok CM, Nogueira RG, Yoo AJ, Leslie-Mazwi TM, Hirsch JA, Stapleton CJ, et al. Transarterial venous sinus occlusion of dural arteriovenous fistulas using ONYX. Interv Neuroradiol 2016;22:711-6. 